\title{
Transcatheter mitral valve implantation (TMVI) using the Edwards FORTIS device
}

Vinayak Bapat ${ }^{1 *}$, FRCS (CTh); Lutz Buellesfeld², MD; Mark D. Peterson ${ }^{3}$, MD; Jane Hancock ${ }^{1}$, FRCP; David Reineke², MD; Chris Buller ${ }^{3}$, MD; Thierry Carrel², MD; Fabien Praz ${ }^{2}$, MD; Ronal Rajani ${ }^{1}$, FRCP; Neil Fam³, MD; Han Kim³, MD; Simon Redwood ${ }^{1}$, FRCP; Christopher Young ${ }^{1}$, FRCS (CTh); Christopher Munns ${ }^{1}$, FRCA; Stephan Windecker², MD; Martyn Thomas ${ }^{1}$, FRCP

1. Department of Cardiothoracic Surgery and Cardiology, St. Thomas' Hospital, London, United Kingdom; 2. Department of Cardiac Surgery and Cardiology, Bern University Hospital, Bern, Switzerland; 3. Department of Cardiac Surgery and Cardiology, St. Michael's Hospital, Toronto, Canada

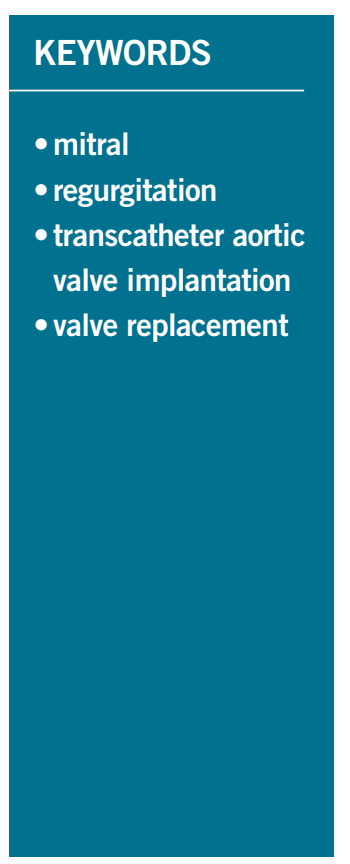

\begin{abstract}
Transcatheter aortic valve implantation (TAVI) has demonstrated the feasibility of treating valvular heart disease with transcatheter therapy. On the back of this success, various transcatheter concepts are being evaluated to treat other valvular disease, especially mitral regurgitation (MR). The concepts currently approved to treat MR replicate surgical mitral valve repair. However, most of them cannot eliminate MR completely. Similar to TAVI, a transcatheter mitral valve implantation may provide a valuable alternative. The FORTIS transcatheter mitral valve (Edwards Lifesciences, Irvine, CA, USA) is a self-expanding device implanted via a transapical approach. We describe our experience and early results in the first five patients treated on compassionate grounds. We also describe the details of the device, selection criteria and technical details of implantation.
\end{abstract}

\footnotetext{
*Corresponding author: Cardiovascular Directorate, 6th Floor, East Wing, St. Thomas' Hospital, Westminster Bridge Road, London, SE1 7EH, United Kingdom.E-mail:vnbapat@yahoo.com
} 


\section{Introduction}

Mitral valve regurgitation (MR) is one of the most common valvular heart diseases in an ageing population and in patients with heart failure ${ }^{1}$. An estimated four million US citizens have moderate to severe $\mathrm{MR}^{2}$. When MR is haemodynamically significant, the treatment options range from medical treatment in asymptomatic patients with normal left ventricular (LV) function to surgical mitral valve repair or replacement ${ }^{3-5}$. Similar to the trend observed in aortic stenosis a decade ago before acceptance of transcatheter aortic valve implantation (TAVI), a large number of patients are not considered suitable for surgery due to the presence of comorbidities which increase the risk of surgery ${ }^{6}$. The Euro Heart Survey has revealed that up to $50 \%$ of symptomatic patients hospitalised with severe MR may not be referred to surgery due to advanced age, comorbidities, and impaired LV function. Advanced age is one of the deterrents for surgery with only $15 \%$ of patients aged greater than 80 years being treated surgically compared to $60 \%$ of patients aged 70 years and younger?

A less invasive surgical option, minimally invasive mitral valve surgery (MIMVS), has become well-established, with an observed reduction in morbidity and mortality in high-risk patients ${ }^{8}$. However, MIMVS techniques rely on cardiopulmonary bypass (CPB) and are often associated with increased duration of operative time. The success of TAVI has opened doors to the possibility of treating a valve lesion without the use of $\mathrm{CPB}$, i.e., on a beating heart through a minimally invasive approach ${ }^{9-12}$. It is therefore not surprising that development has taken place in the field of transcatheter treatments for mitral valve regurgitation. The transcatheter treatments currently available for commercial use or under evaluation are predominantly those which attempt to replicate a mitral repair ${ }^{13-16}$. The MitraClip ${ }^{\mathrm{TM}}$ system (Abbott Vascular Inc., Menlo Park, CA, USA) was one of the first transcatheter devices to be commercialised (2008) and received FDA approval in 2013. It is based on the principle of Alfieri's edgeto-edge surgical repair ${ }^{13}$. Similarly, devices which can perform a direct or indirect annuloplasty and insertion of new chordae are undergoing evaluation ${ }^{14-16}$. One of the drawbacks of these devices is failure to eliminate MR completely.
Transcatheter mitral valve implantation (TMVI), on the other hand, may provide a valuable alternative by providing a new valve, which when implanted correctly eliminates MR completely while providing a sufficient orifice area. When compared to the aortic valve, the mitral valve has a larger and non-circular saddle-shaped annulus, a complex subvalvular apparatus and potential for left ventricular outflow tract (LVOT) obstruction. These attributes, along with the absence of calcification in MR and high mitral transvalvular gradients, have made development of a transcatheter mitral valve (TMV) device challenging.

The first experimental off-pump TMV implantation via the left atrium was reported by Ma et al in $2005^{17}$. In early 2014, the firstin-human percutaneous TMVI with the FORTIS TMV was performed at the Guy's and St. Thomas' Hospital, London. Within a few months, four additional implants were performed in Guy's and St. Thomas' Hospital, Bern University Hospital, Bern, and St. Michael's Hospital, Toronto. The aim of this paper is to provide an overview of the FORTIS TMV and its delivery system, patient selection, important procedural steps, early results and future direction.

\section{Edwards FORTIS TMV}

The Edwards FORTIS TMV is made of a cloth-covered, selfexpanding nitinol stent with three bovine pericardial leaflets. At present only the $29 \mathrm{~mm}$ size valve is available for clinical use (Figure 1A, Figure 1B).

The FORTIS TMV consists of three main components:

1. A central valve body;

2. Paddles;

3. An atrial flange.

The central valve body is a cylindrical central portion of $29 \mathrm{~mm}$ diameter, which harbours the three leaflets. The leaflets are similar to those used in other surgical and transcatheter valves manufactured by Edwards Lifesciences and are sutured to the stent. The leaflets are treated with the GLX tissue treatment technology (Edwards Lifesciences), which allows for packaging and sterilisation in a dry condition and is designed to provide additional protection against calcification. The outside of the stent is cloth-covered

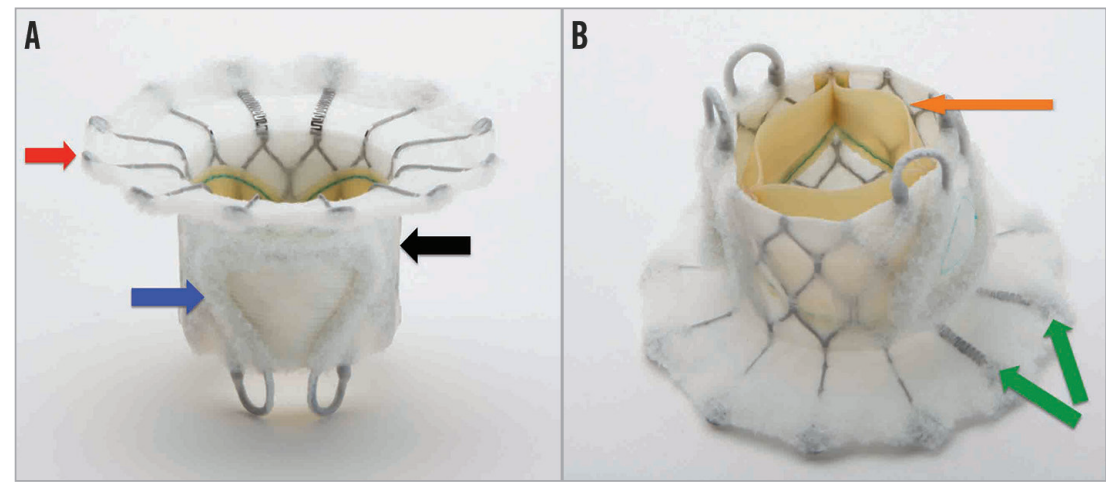

Figure 1. FORTIS transcatheter mitral valve. A) Side profile of the FORTIS valve highlighting the atrial flange (red arrow), body of the FORTIS valve (black arrow) and one of the two paddles (blue arrow). B) Side profile of the FORTIS valve highlighting the bovine pericardial leaflets (orange arrow) and the flexible struts (green arrows), which align with the A2 segment of the mitral valve. 
to prevent mitral leaflet injury and provide a platform for tissue ingrowth after implantation.

Two symmetric paddles are attached to the outflow of the central valve body. The paddle structure is designed in such a way that they can be deflected away from the central body to capture the mitral leaflets and then actuated towards the central body and tightened to secure the leaflets between the FORTIS valve body and paddles. The paddles serve to anchor the FORTIS TMV to the native leaflets and valve. The paddles are also covered with fabric, which has a similar function to the cloth covering the valve body.

The atrial flange forms the third part of the device and is positioned at the inflow portion of the valve body. It is made of multiple nitinol struts. Two of the struts that are aligned to the A2 segment of the mitral valve anatomy are more flexible than the rest. The purpose of the flexible atrial struts in this area is to avoid interference with the aortic valve function. The atrial flange is also covered with fabric. The atrial flange is positioned flush with the base of the left atrium and becomes endothelialised due to tissue ingrowth.

\section{Delivery system and accessories}

The Edwards FORTIS TMV is implanted through a transapical (TA) approach using a dedicated FORTIS mitral transcatheter sheathless delivery system (Figure 2A). The delivery system is $42 \mathrm{Fr}$ in outer diameter. The delivery system has a nose cone tip to facilitate entry into the LV and also holds the atrial flange. The main shaft is made of a harness and two sheaths which hold the central valve body and the flange. The main handle has two intuitive knobs which control the movements of the inner and outer sheaths, which in turn help cover and uncover parts of the FORTIS device (Figure 2B). At the end of the handle is a dedicated knob to control the paddles. The delivery system has a central lumen for a guidewire and has multiple radiopaque markers which assist positioning and deployment of the device under fluoroscopy and echocardiography.

\section{Patient screening}

High surgical risk patients with severe mitral regurgitation and NYHA Class II symptoms or greater were screened by a multidisciplinary Heart Team, consisting of two cardiothoracic surgeons and two cardiologists, as potential candidates for a transcatheter mitral valve replacement. Potential candidates underwent screening for the FORTIS valve after being turned down for conventional mitral valve replacement by two surgeons specialising in mitral valve surgery. Patients were discussed in the multidisciplinary meeting before the screening process was initiated. Additionally, patients who were screened for the FORTIS valve were considered poor candidates for the MitraClip based on anatomical findings.

All patients underwent a comprehensive preoperative evaluation, including a coronary angiogram, transoesophageal echocardiogram (TEE) and ECG-gated cardiac CT to define additional anatomic inclusion and exclusion criteria. The $29 \mathrm{~mm}$ FORTIS TMV is suitable for patients with a native annular diameter (A2P2) distance measuring between 30 and $44 \mathrm{~mm}$, a posterior leaflet length of $>0.5 \mathrm{~cm}$ and an anterior leaflet length $<2.3 \mathrm{~cm}$, as assessed by echo. P2 leaflet prolapse, mitral regurgitation that is predominantly commissural and a small left ventricle are contraindications to the FORTIS valve (Table 1 and Table 2). The subvalvular apparatus is assessed by reconstructed CT images to determine the anatomy of the papillary muscles and chordae. Unfavourable anatomy, which may prevent adequate leaflet capture and advancement of the paddles of the FORTIS device to the ventricular side of the mitral annulus, includes irregular papillary muscle head branching, fused papillary muscle heads, chordae extending to the subannular groove of the mitral valve and large strut chordae. The LVOT clearance is also calculated to predict the possibility of LVOT obstruction after implantation of the FORTIS TMV.

\section{Procedural steps}

The procedure is performed in a hybrid theatre or a catheterisation laboratory under general anaesthesia. The procedure is performed under transeosophageal echocardiography (TEE) guidance and is aided by fluoroscopy.

\section{ACCESS}

The LV apex is located with transthoracic echocardiography. This is important as most patients have an enlarged left ventricle. Surface echocardiography is also performed once the apex is exposed so as to

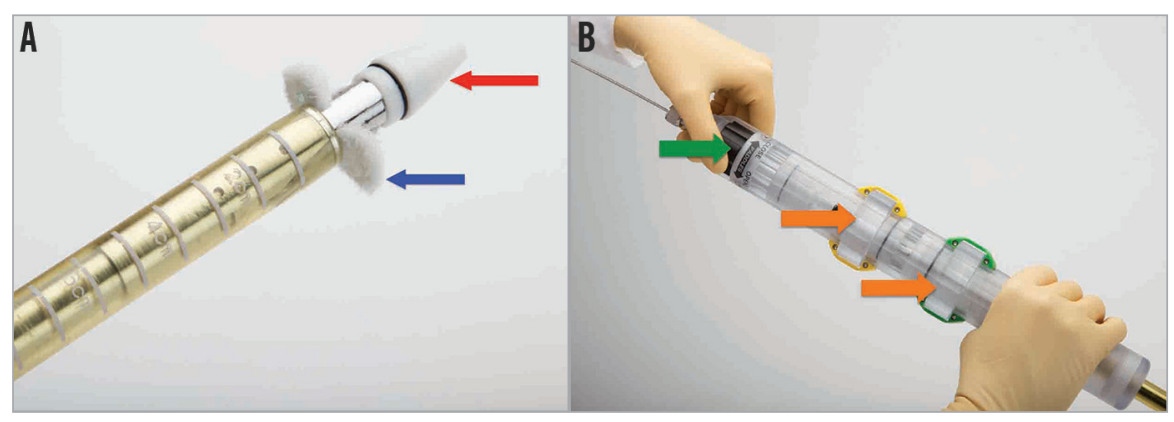

Figure 2. FORTIS delivery system. A) Sheathless delivery system with a nose cone at the tip (red arrow). Partial unsheathing leads to uncovering of the paddles (blue arrow). B) Handle of the delivery system with two wheels/knobs (orange arrows), which control sheathing and unsheathing of the valve body and paddles. The green arrow points to the wheel/knob, which controls opening and closing of the paddles. 
locate the best spot for purse-string placement. Two purse-strings are placed onto the chosen spot similar to a transapical approach for TAVI.

\section{WIRE PLACEMENT}

After gaining transapical access with a standard puncture needle, a regular wire is placed in the left atrium and exchanged for a stiff guidewire such as an Amplatz Super Stiff ${ }^{\text {TM }}$ guidewire (Boston Scientific, Natick, MA, USA). TEE and fluoroscopy are used to confirm accurate placement of the guidewire free from chordal entanglement. This is one of the most important steps in the procedure.

\section{DELIVERY SYSTEM INSERTION}

The apex can be dilated with two dedicated dilators prior to the insertion of the delivery system. The delivery system with the crimped FORTIS TMV is introduced into the LV over the stiff wire until the nose cone is at the level of the mitral annulus.

\section{VALVE DEPLOYMENT}

The paddles are partially unsheathed, and paddle orientation perpendicular to the mitral coaptation line is confirmed by TEE. The paddles are then opened up to 45 degrees and the delivery system is advanced to capture the mitral leaflets, until the tips of the paddles reach the annular plane (Figure $3 \mathbf{A}$ ). This can only be achieved by placing the paddles in the A2-P2 area. Once the leaflet capture is confirmed, the device is partially unsheathed into the native mitral valve annulus. Additional assessments are performed to ensure device positioning is optimal and native leaflet capture is maintained before release of the atrial flange. Until this step, the device is completely recapturable and repositionable. The atrial flange is deployed by pushing the nose cone forward into the left atrium (Figure 3B). Final echo assessments are performed and, if the position is satisfactory, the paddles are closed over the partially unsheathed device and the device is then completely released, allowing a full expansion of the FORTIS TMV (Figure 3C). Rapid pacing is not necessary as throughout the entire procedure normal blood flow is maintained.

\section{POST-DEPLOYMENT}

TEE assessment is performed for stability and function of the valve (Figure 4). Once the delivery system is removed, the apical access site is closed and then the chest is closed in a routine manner.

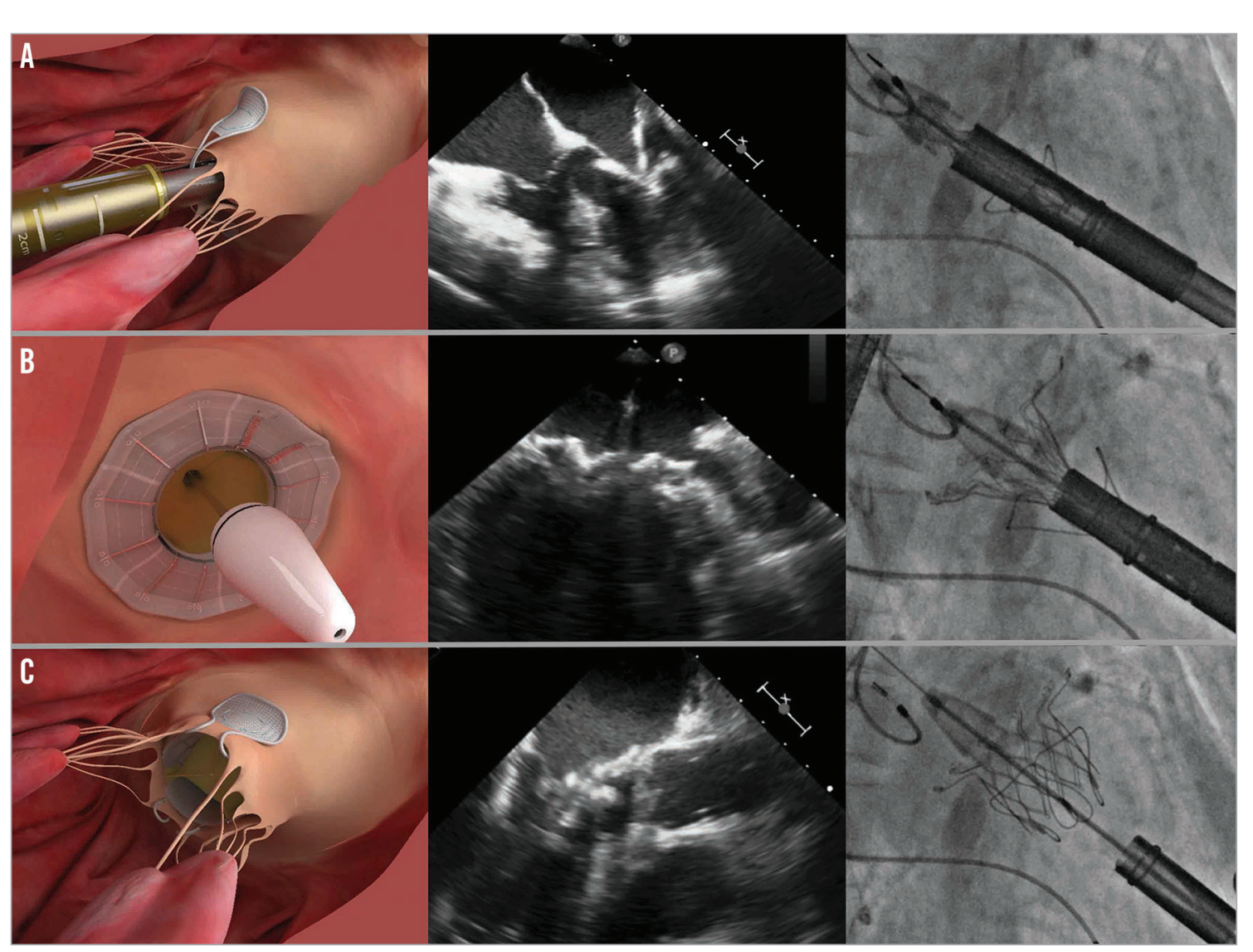

Figure 3. Important steps in FORTIS valve implantation illustrated with a picture, TEE image and fluoroscopic image. A) Leaflet capture: the paddles are seen outside both of the leaflets. Closing will result in trapping the leaflets between the paddles and the valve body. B) Release of the atrial flange. Once leaflet capture is confirmed, the atrial flange is released by pushing the nose cone forward. C) Valve deployment: once the paddles are closed over the body to anchor the device securely, the valve is released by unsheathing the ventricular portion. 


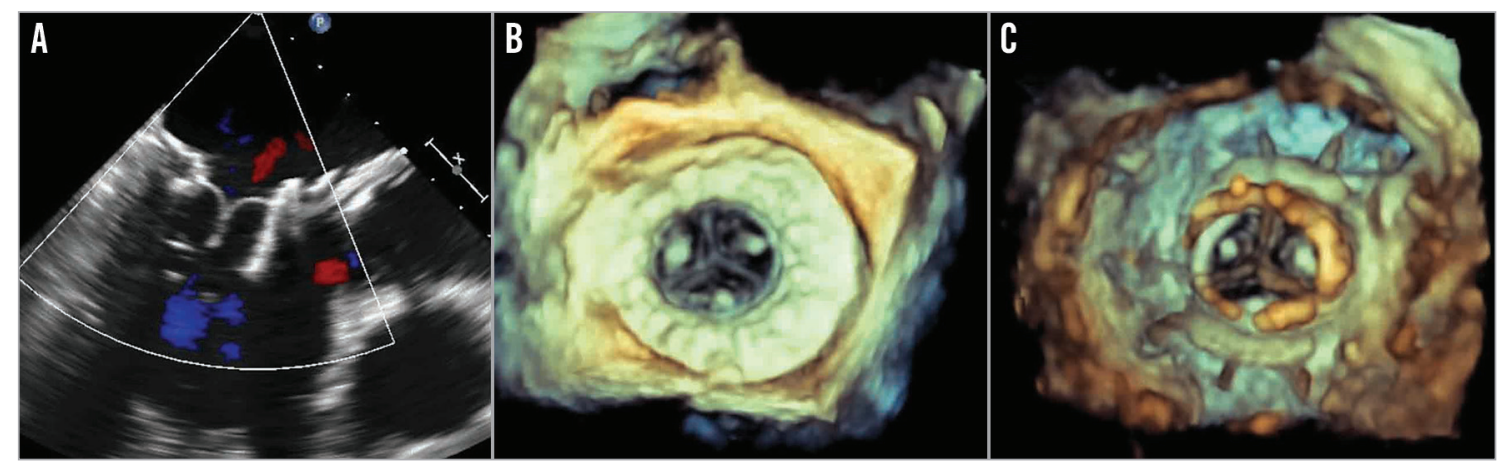

Figure 4. Assessment of the FORTIS valve function after implantation. A) Two-dimensional echocardiography demonstrates well-seated and functioning FORTIS device. B) 3D TEE assessment demonstrates excellent valve function from the atrial side. Atrial flange is clearly seen. C) 3D TEE assessment demonstrates well-functioning leaflets from the ventricular side.

\section{Early results}

All cases were performed on compassionate grounds and not within a trial protocol. These patients were considered suitable for the therapy only when considered unsuitable for conventional approved treatments such as surgery. Patients were discussed in departmental multidisciplinary meetings. Detailed discussions were held with the patients before screening procedures were initiated. After the screening, suitability for the $29 \mathrm{~mm}$ FORTIS TMV was evaluated and, once considered suitable, approval was obtained from the local hospital committees and regulatory bodies in the respective countries. Important points, procedural details and early outcomes are discussed below.

\section{PATIENT 1}

Patient 1 had an EF of $15-20 \%$ with a large heart. The LV apex was located in the mid axillary line. The TEE images were difficult, especially visualisation of the PML. Fluoroscopic implant angles, which could help the implantation procedure, could not be obtained, and hence the procedure was performed with echo guidance and only aided by fluoroscopy. Implantation was successful with minimal MR in the posteromedial commisure. The patient was extubated on the table. Recovery was slow due to persistent heart failure. The patient was discharged on day 15. The 30-day follow-up assessment revealed marginal improvement despite minimal MR. The patient was readmitted on day 37 with signs and symptoms of heart failure. Despite aggressive treatment he passed away on day 76 .

\section{PATIENT 2}

Patient 2 suffered from chronic renal failure with functioning transplant, heavily calcified coronary arteries and the presence of LV apical aneurysm. The procedure was technically difficult because the purse-string access could not be placed at an ideal spot due to an apical aneurysm. This proved critical, as capturing AML and PML simultaneously was challenging. During the procedure, visualisation of the PML capture was difficult to assess. However, a satisfactory result was obtained after the implant with trace MR. The patient was extubated on the table. The patient developed acute renal failure on day 1 and, because of the prior renal transplant, there was a delay in commencing filtration. She went into pulmonary oedema on day 2 and was re-intubated. TOE demonstrated increasing MR, and the posterior paddle was seen to be displaced in the left atrium. Despite aggressive management she passed away on day 4. Autopsy findings confirmed loss of capture of the posterior leaflet with partial displacement of the device in the left atrium (Figure 5).

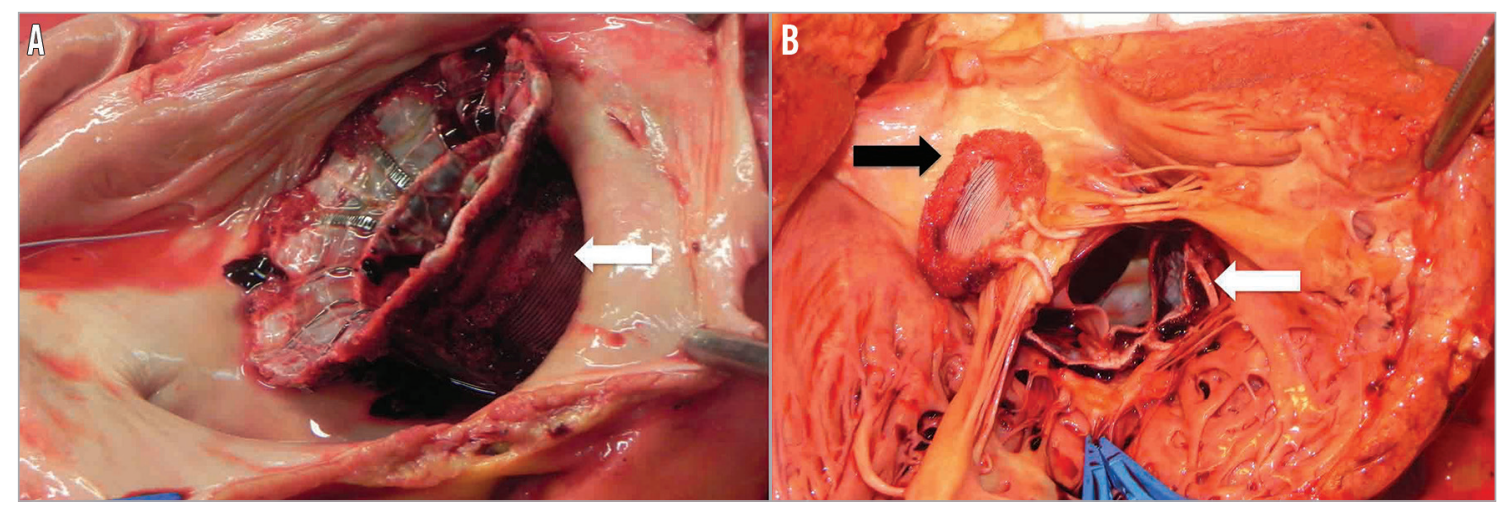

Figure 5. Post mortem findings in patient 2. A) Atrial view confirmed partial displacement of the FORTIS device into the left atrium. White arrow points to the posterior paddle seen inside the PML. B) Ventricular view confirmed partial displacement because of failure of the posterior paddle (white arrow) to capture the PML (blue forceps). Anterior paddle was securely anchored (black arrow). 


\section{PATIENT 3}

Patient 3 had prior CABG with patent grafts and severe MR. Although the apical access was challenging due to COPD and large BMI, the procedure was uneventful. The size of the ventricle and the apical puncture achieved near the true apex both contributed to excellent access to the mitral valve and feasible capture of both leaflets. The procedure was TEE-guided due to the patient's large size and difficult fluoroscopic projections. There was no evidence of residual mitral regurgitation. The patient was extubated on the table and was discharged on day 6. The 30-day follow up assessment demonstrated satisfactory device function and improvement in the six-minute walk test (from 135 metres to 215 metres). The patient continues to improve slowly.

\section{PATIENT 4}

Patient 4 had prior CABG with patent grafts and similar LV anatomy to patient 3 . The procedure was TEE-guided and implantation was smooth with an excellent result (Figure 6A). The patient was extubated on the table and discharged on day 9. The patient was preoperatively on rivaroxaban, which was replaced with coumadin postoperatively. INR was therapeutic on the day of discharge. He was readmitted on day 15 with cardiac decompensation, abdominal pain and a systemic inflammatory response syndrome. Echocardiography demonstrated reduced leaflet mobility of two of the three leaflets of the FORTIS TMV with high gradients (Figure 6B). INR was 3.5. Antibiotics and additional heparin were commenced, but rapid deterioration led to death on day 15. Post mortem examination was not carried out due to the family's wishes.

\section{PATIENT 5}

Patient 5 had known coronary artery disease with two prior infarctions, and PCI was performed on both occasions. There was significant respiratory risk due to bronchiectasis, frequent pneumonia and prior thoracotomy and right lung decortication. The procedure was TEE-guided and was uneventful with an excellent result. There was no evidence of residual MR. The patient was discharged home on day 6 and continues to do well with improved effort tolerance.

\section{ANTICOAGULATION REGIMEN}

Unless contraindicated, all patients were commenced on dual antiplatelet therapy and anticoagulation on day 1 . Warfarin was preferred in all patients. Target INR was $>2.5$. After three months, the regimen was changed to single antiplatelet therapy and warfarin.

\section{Discussion}

Success and adaptation of TAVI for the treatment of aortic valve disease has led to interest in developing transcatheter therapies for other valves, especially the mitral valve. Initial concepts in treating MR were predominantly focused on replicating surgical mitral valve repair ${ }^{9-12}$.

The device with the largest amount of clinical experience is the MitraClip ${ }^{\mathrm{TM}}$ system. MitraClip replicates the Alfieri stitch, which involves suturing the two leaflets of the mitral valve together in the A2-P2 region ${ }^{13}$. The procedure is performed via the transseptal approach under echocardiography guidance. MitraClip obtained CE mark in 2008 and was approved by the FDA in October 2013 for patients with significant symptomatic degenerative MR who are at prohibitive risk for mitral valve surgery. Approval was based on the results of the Endovascular Valve Edge-to-Edge Repair Study (EVEREST II) and other registry data ${ }^{18,19}$. In the EVEREST II study, two-year results showed that percutaneous mitral valve edgeto-edge repair was less effective in reducing MR than surgery but was associated with superior safety and similar improvements in clinical outcomes. Trials studying the role of the MitraClip system in patients with symptomatic functional MR (RESHAPE, COAPT) are still ongoing. However, MitraClip may not be suitable for all patients with MR, as it reduces the mitral valve orifice area and does not eliminate MR completely ${ }^{18,19}$.

The other percutaneous technologies, which are in early stages of development, such as those employing the concepts of annuloplasty, chordal implantation and LV remodelling, have either shown limited efficacy and/or are still in a very premature phase of development ${ }^{14-16}$. As transcatheter therapies are trying to replicate surgical repair, it can be anticipated that these repair technologies will have limited applicability, as a surgical repair is usually a combination

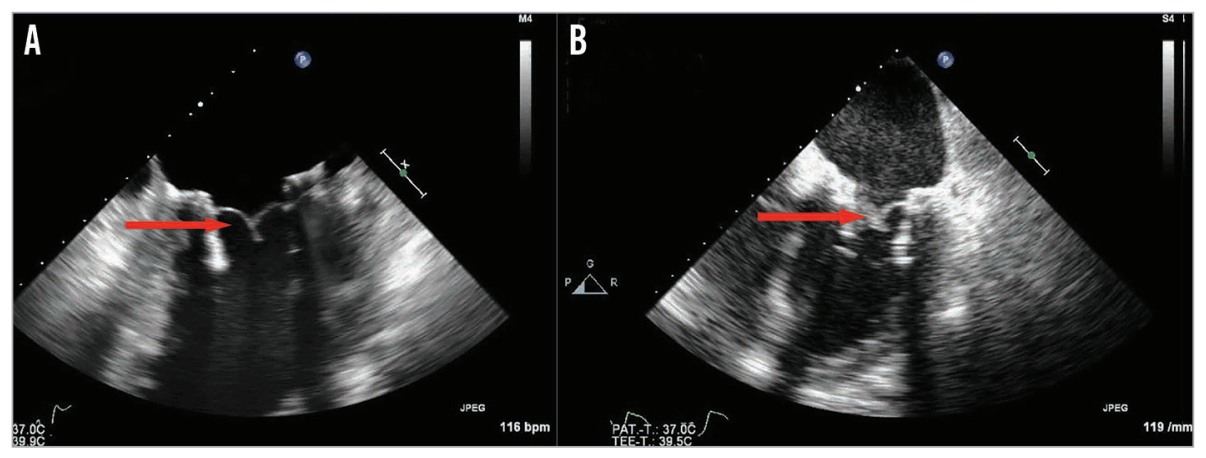

Figure 6. Echocardiographic findings in patient 4. A) TEE immediately post-implantation demonstrates well-functioning leaflets and no evidence of thickening (red arrow). B) TEE findings on readmission demonstrate thickening of two of the three leaflets (red arrow). It was unclear if this was thrombus lining or inflammatory reaction. 
Table 1. Echocardiography inclusion criteria to determine suitability of the patient for a $29 \mathrm{~mm}$ FORTIS valve implantation.

\begin{tabular}{|l|c|c|c|}
\hline \multicolumn{1}{|c|}{ Dimension } & Sizing feature/potential adverse effect & Phase & Target range \\
\hline A2 P2 distance & Valve body diameter & Systole & $\geq 3.0 \mathrm{~cm}$ \\
\hline A2 P2 distance & & Diastole & $\leq 4.4 \mathrm{~cm}$ \\
\hline AML length from the hinge point & Valve body diameter & NA & $<2.3 \mathrm{~cm}$ \\
\hline PML length & Inability to capture leaflets & NA & $>0.5 \mathrm{~cm}$ \\
\hline PML: posterior mitral leaflet & &
\end{tabular}

of annuloplasty, leaflet remodelling and/or chordal replacements ${ }^{18}$. Hence, transcatheter mitral valve replacement (TMVI) may be another useful alternative to treat mitral regurgitation.

TMVI, however, poses unique challenges. Some of these are discussed below.

1. Absence of a calcified valve: during TAVI, the calcified aortic valve provides stability. With mitral regurgitation the valve is not calcified although part of the annulus may be, hence it requires a different mechanism for anchoring. Calcification also serves as a fluoroscopic landmark. These are absent during TMVI. Hence, TMVI is predominantly an echocardiographyguided procedure and is dependent on intraoperative echo imaging. This became obvious during our experience as we switched the focus from fluoroscopy to echocardiography from patient 2 , and the last two procedures were predominantly performed under echocardiography guidance.

2. Larger valve size: the mitral valve area is much larger than the aortic valve area and hence a larger TMVI device is needed. This also means a larger crimped diameter and larger delivery system calibre.

3. Complex structure: the mitral valve has a subvalvar apparatus, two unequal leaflets and a non-circular annulus. Also, closing pressures are higher, i.e., systolic. These factors pose a unique challenge in designing the transcatheter prosthesis.

4. Possibility of LVOT obstruction: the anterior mitral leaflet (AML) acts as a partition between the left ventricular inflow and LVOT. After TMVI, the AML may be displaced to a certain degree into the LVOT. This is dependent on various anatomical and device-related factors. A large septal bulge and small ventricular size may further compound this problem.

A first-in-man experience can be challenging as one has to transfer the knowledge gained from animal models to the human anatomy and this may not be easy. In relation to FORTIS implantation, one of the examples is use of fluoroscopy during the procedure. In the animal model, it was possible to obtain necessary angles perpendicular to the mitral annulus to use certain features on the delivery system to facilitate implantation. These angles, however, could not be obtained in any of the five patients. Hence, we had to modify the procedure in terms of reliance on TEE rather than fluoroscopy. This reflected partially on the procedure times in the first three patients (Figure 7). By the time the fourth patient underwent the procedure, the sequence of steps and checklists had been standardised and hence the procedure was smoother and the duration much reduced.

As cases were performed on compassionate grounds, the patients were high risk. Also, our initial focus was to confirm suitability of the mitral anatomy to the FORTIS device but, after the first two

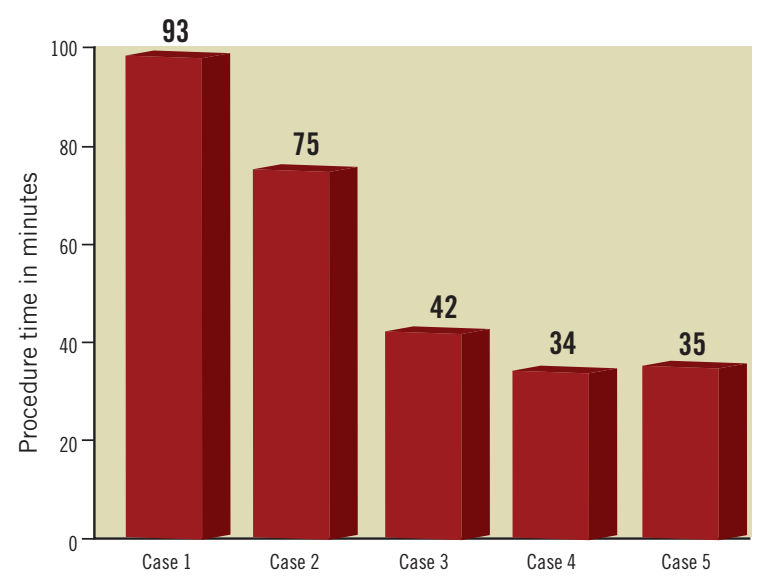

Figure 7. Duration of FORTIS implantation in minutes. Graph demonstrates time from puncture to deployment of the FORTIS device. Significant reduction in the duration of FORTIS implantation is evident in patients 4 and 5.

Table 2. CT screening and anatomical criteria measured to determine suitability for a $29 \mathrm{~mm}$ FORTIS valve.

\begin{tabular}{|c|c|c|c|}
\hline Dimension & Sizing feature/potential adverse effect & Phase & Target range \\
\hline LA minor diameter & \multirow{2}{*}{ Atrial flange diameter } & Systole & $\leq 52 \mathrm{~mm}$ \\
\hline LA minor diameter & & Diastole & $\geq 38 \mathrm{~mm}$ \\
\hline LVOT width & \multirow{3}{*}{$\begin{array}{l}\text { LVOT obstruction } \\
\text { damage to ventricular wall }\end{array}$} & \multirow{3}{*}{ Systole } & N/A \\
\hline Aorta to device plane angle & & & $\geq 90$ degrees \\
\hline Calculated LVOT clearance & & & $>0 \mathrm{~mm}$ \\
\hline LV diameter at papillary muscle plane & Damage to ventricular wall & Systole & $>32 \mathrm{~mm}$ \\
\hline LA height & Delivery system clearance & Diastole & $>30 \mathrm{~mm}$ \\
\hline
\end{tabular}


patients, it became clear that we also needed to analyse for optimal LV geometry and access point. This was reflected in the following three patients. Patients, such as patient 1 , with an enlarged left ventricle and low EF $(<20 \%)$ may not benefit from TMVI and in fact this may hasten the inevitable refractory heart failure due to complete elimination of MR. In future, we may need to evaluate these patients for functional reserve if contemplating TMVI ${ }^{19}$.

Three patients survived beyond 30 days, and two patients were alive at the time of reporting (patients 3 and 5). The cause of death in patient 1 was intractable heart failure despite a good procedural result. Patient 2 demonstrated the importance of an optimal access point and complete capture of both mitral leaflets. Partial capture of the PML resulted in a satisfactory result immediately after implantation but, due to higher closing pressures, also resulted in upward displacement of the device and severe MR. The clinical picture and events leading to the death of patient 4 could not be explained completely. The clinical picture was one of cardiac decompensation with rapid deterioration and a systemic inflammatory response syndrome within a very short period of time after an uneventful recovery and discharge. Echocardiography resembled valve thrombosis but the INR was always therapeutic. Blood cultures were negative throughout the admission. A post mortem was not carried out due to family wishes and hence the exact cause of death remains unknown.

Anticoagulation management will be important after TMVI and our protocol has been to commence subcutaneous heparin as soon as possible. Triple therapy consisting of dual antiplatelet and oral anticoagulation with vitamin-K antagonist was started on day 1 in all patients. The aim is to continue this regimen for at least 90 days and then switch to a single antiplatelet agent and vitamin$\mathrm{K}$ antagonist. Prevention of thromboembolic complications with an antithrombotic regimen associated with low bleeding risk will be the key priority in the management of TMVI patients.

To conclude, patients 3 and 5 provide proof-of-principle in successfully treating MR in high-risk patients not suitable for currently available treatments including surgery. Analysing procedural difficulties led to successful modification of procedural steps, and the screening process and additional experience will continue to allow refinement of the procedure. The complex structure of the mitral valve will pose a stiffer challenge when compared to TAVI, but the experience gained from the physicians and the industry from the development of TAVI will facilitate development of TMVI. We expect a similar evolution as was observed with the introduction of TAVI, and TMVI may eventually become a viable therapeutic alternative to surgical valve repair/replacement in the future.

\section{Conflict of interest statement}

V. Bapat is a consultant for Edwards Lifesciences, Medtronic Inc and St. Jude Medical. S. Redwood is a consultant for Edwards Lifesciences. M. Thomas is a consultant for Edwards Lifesciences and St. Jude Medical. C. Young and M. Peterson are consultants for Edwards Lifesciences. The other authors have no conflicts of interest to declare.

\section{References}

1. Benjamin MM, Smith RL, Grayburn PA. Ischaemic and functional mitral regurgitation in heart failure: natural history and treatment. Curr Cardiol Rep. 2014;16:517.

2. De Backer O, Piazza N, Banai S, Lutter G, Maisano F, Herrmann HC, Franzen OW, Søndergaard L. Percutaneous transcatheter mitral valve replacement: an overview of devices in preclinical and early clinical evaluation. Circ Cardiovasc Interv. 2014;7:400-9.

3. Messas N, Samet H, Brocchi J, Billaud P, Kremer H, Jesel L, Ohlmann P, Morel O. Mitral valve surgery for transient severe mitral regurgitation: An alternative to medical treatment? Int $J$ Cardiol. 2014;175:e40-2.

4. Weiner MM, Hofer I, Lin HM, Castillo JG, Adams DH, Fischer GW. Relationship among surgical volume, repair quality and perioperative outcomes for repair of mitral insufficiency in a mitral valve reference center. J Thorac Cardiovasc Surg. 2014 Apr 23. pii: S0022-5223(14)00514-5. doi: 10.1016/j.jtcvs.2014.04.040. [Epub ahead of print].

5. Acker MA, Parides MK, Perrault LP, Moskowitz AJ, GelijnsAC, VoisineP, SmithPK, Hung JW, BlackstoneEH,Puskas JD, Argenziano M, Gammie JS, Mack M, Ascheim DD, Bagiella E, Moquete EG, Ferguson TB, Horvath KA, Geller NL, Miller MA, Woo YJ, D'Alessandro DA, Ailawadi G, Dagenais F, Gardner TJ, O'Gara PT, Michler RE, Kron IL; CTSN. Mitral-valve repair versus replacement for severe ischemic mitral regurgitation. $N$ Engl $J$ Med. 2014;370:23-32.

6. Philip F, Athappan G, Tuzcu EM, Svensson LG, Kapadia SR. MitraClip for severe symptomatic mitral regurgitation in patients at high surgical risk: a comprehensive systematic review. Catheter Cardiovasc Interv. 2014 Jun 6. doi: 10.1002/ccd.25564. [Epub ahead of print].

7. Niemien MS, Brutsaert D, Dickstein K, Drexler H, Follath F, Harjola VP, Hochadel M, Komajda M, Lassus J, Lopez-Sendon JL, Ponikowski P, Tavazzi L; EuroHeart Survey Investigators; Heart Failure Association, European Society of Cardiology. EuroHeart Failure Survey II (EHFS II): a survey on hospitalized acute heart failure patients: description of population. Eur Heart J. 2006;27: 2725-36.

8. Goldstone AB, Joseph Woo Y. Minimally invasive surgical treatment of valvular heart disease. Semin Thorac Cardiovasc Surg. 2014;26:36-43.

9. Généreux P, Head SJ, Wood DA, Kodali SK, Williams MR, Paradis JM, Spaziano M, Kappetein AP, Webb JG, Cribier A, Leon MB. Transcatheter aortic valve implantation 10-year anniversary: review of current evidence and clinical implications. Eur Heart J. 2012;33:2388-98.

10. Elmariah S, Palacios IF, McAndrew T, Hueter I, Inglessis I, Baker JN, Kodali S, Leon MB, Svensson L, Pibarot P, Douglas PS, Fearon WF, Kirtane AJ, Maniar HS, Passeri JJ; PARTNER Investigators. Outcomes of transcatheter and surgical aortic valve replacement in high-risk patients with aortic stenosis and left ventricular dysfunction: results from the Placement of Aortic 
Transcatheter Valves (PARTNER) trial (cohort A). Circ Cardiovasc Interv. 2013;6:604-14.

11. Wendler O, Walther T, Schroefel H, Lange R, Treede H, Fusari M, Rubino P, Thomas M; SOURCE investigators. Transapical aortic valve implantation: mid-term outcome from the SOURCE registry. Eur J Cardiothorac Surg. 2013;43: 505-11.

12. Beckmann A, Hamm C, Figulla HR, Cremer J, Kuck KH, Lange R, Zahn R, Sack S, Schuler GC, Walther T, Beyersdorf F, Böhm M, Heusch G, Funkat AK, Meinertz T, Neumann T, Papoutsis K, Schneider S, Welz A, Mohr FW; GARY Executive Board. The German Aortic Valve Registry (GARY): a nationwide registry for patients undergoing invasive therapy for severe aortic valve stenosis. Thorac Cardiovasc Surg. 2012;60: 319-25.

13. Mauri L, Foster E, Glower DD, Apruzzese P, Massaro JM, Herrmann HC, Hermiller J, Gray W, Wang A, Pedersen WR, Bajwa T, Lasala J, Low R, Grayburn P, Feldman T; EVEREST II Investigators. 4-year results of a randomized controlled trial of percutaneous repair versus surgery for mitral regurgitation. $J \mathrm{Am}$ Coll Cardiol. 2013;62:317-28.
14. Chiam PT, Ruiz CE. Percutaneous transcatheter mitral valve repair: a classification of the technology. JACC Cardiovasc Interv. 2011;4:1-13.

15. Cubeddu RJ, Palacios IF. Percutaneous techniques for mitral valve disease. Cardiol Clin. 2010;28:139-53.

16. Seeburger J, Rinaldi M, Nielsen SL, Salizzoni S, Lange R, Schoenburg M, Alfieri O, Borger MA, Mohr FW, Aidietis A. Offpump transapical implantation of artificial neo-chordae to correct mitral regurgitation: the TACT Trial (Transapical Artificial Chordae Tendinae) proof of concept. J Am Coll Cardiol. 2014;63:914-9.

17. Ma L, Tozzi P, Huber CH, Taub S, Gerelle G, von Segesser LK. Double-crowned valved stents for off-pump mitral valve replacement. Eur J Cardiothorac Surg. 2005;28:194-8.

18. Castillo JG, Milla F, Anyanwu AC, Adams AC. Video-atlas on minimally invasive mitral valve surgery-The David Adams technique. Ann Cardiothorac Surg. 2013;2:828-32.

19. Lio A, Miceli A, Varone E, Canarutto D, Di Stefano G, Della Pina F, Gilmanov D, Murzi M, Solinas M, Glauber M. Mitral valve repair versus replacement in patients with ischaemic mitral regurgitation and depressed ejection fraction: risk factors for early and midterm mortality. Interact Cardiovasc Thorac Surg. 2014;19:64-9. 